\title{
Two-channel Near-infrared Spectroscopic Analysis of Association of Paranoia Symptoms with Prefrontal Activation
}

\author{
Kazuki Hirao \\ Department of Occupational Therapy, School of Health Science and Social Welfare, Kibi International University, Okayama, Japan
}

\begin{abstract}
Objective: The relationship between paranoia symptoms and underlying prefrontal cortex mechanisms among healthy subjects was analyzed using near-infrared spectroscopy.

Methods: Seventy-eight healthy subjects were assessed for paranoia symptoms using the Japanese version of the Paranoia Checklist. Changes in hemoglobin concentrations were assessed using 2-channel near-infrared spectroscopy on the surface of the prefrontal cortex while subjects performed a verbal fluency test.

Results: Changes in the concentration of oxygenated hemoglobin in the prefrontal cortex during a verbal fluency test did not correlate with the Japanese version of the Paranoia Checklist.

Conclusion: Our findings show that the symptoms of paranoia do not negatively affect the prefrontal cortex function among healthy subjects.
\end{abstract}

KEY WORDS: Paranoia disorders; Prefrontal cortex; Near-infrared spectroscopy.

\section{INTRODUCTION}

Paranoia is a common symptom of schizophrenia. ${ }^{1,2)}$ The results of a functional magnetic resonance imaging (fMRI) study suggest that patients with a profile of paranoia are characterized by poor social function and insight. ${ }^{3)}$ Freeman et al. ${ }^{4)}$ reported that paranoia symptoms observed in members of a clinical group were present in members of nonclinical groups, suggesting the continuity of symptoms. Moreover, sensitivity to paranoid ideation in nonclinical populations is present in $40 \%$ of subjects who frequently express the view that negative comments are being circulated about them, and approximately $20 \%$ of this population frequently expresses paranoid thoughts about being observed or followed. ${ }^{4)}$ The symptoms of paranoia are observed in nonclinical populations at high frequency. ${ }^{5,6)}$ Accordingly, clinical intervention is required for clinical as well as nonclinical populations.

Received: June 29, 2014 / Revised: August 27, 2014

Accepted: September 20, 2014

Address for correspondence: Kazuki Hirao, OT, PhD Department of Occupational Therapy, School of Health Science and Social Welfare, Kibi International University, 8 Iga-machi Takahashi, Okayama 716-8508, Japan

Tel: +81-866-22-4044, Fax: +81-866-22-4044

E-mail: hirao-k@kiui.ac.jp
To understand the cortical (i.e., prefrontal cortex $[\mathrm{PFC}])$ mechanisms of the pathogenesis of the symptoms of paranoia, it is necessary to investigate nonclinical populations. However, whether the symptoms of paranoia in the nonclinical population are associated with brain activation is unknown. The availability of near-infrared spectroscopy (NIRS) allows noninvasive measurement of cortical activation under normal conditions while a subject performs verbal fluency tasks such as the Verbal Fluency Test (VFT). Because the changes in [oxy-Hb] are assumed to more directly reflect cognitive activation than those in [deoxy- $\mathrm{Hb}]$, the results of a previous study have suggested a more significant correlation with blood oxygenation level-dependent signals compared with those measured with fMRI. ${ }^{7)}$ Therefore, this study aimed to assess the relationship between the symptoms of paranoia and the underlying PFC activity in healthy subjects using NIRS.

\section{METHODS}

\section{Subjects}

All 78 subjects were right-handed and healthy and were undergraduate students attending Kibi International University in Japan. This study was conducted as per the ethical principles laid down in the Declaration of Helsinki.

(c) This is an Open-Access article distributed under the terms of the Creative Commons Attribution Non-Commercial License (http://creativecommons.org/licenses/by-nc/3.0) which permits unrestricted non-commercial use, distribution, and reproduction in any medium, provided the original work is properly cited. 
All subjects were informed of the purpose and procedures of the study, and they provided their written consent. This study was approved by the Institutional Review Board of Kibi International University.

\section{Procedure}

\section{Symptoms of paranoia}

Symptoms of paranoia were determined using the Japanese version of the Paranoia Checklist (JPC), ${ }^{8,9}$ which was translated into Japanese. ${ }^{4)}$ This scale was developed to assess paranoid ideation in a nonclinical population and comprises a self-rating scale for nine questionnaires measured on a five-point scale with anchoring values of 1 and 5. This scale measures the dimensions of frequency, conviction, and distress. A high score indicates a high level of frequency (How often have you had the thought?), conviction (How strongly do you believe it?), and distress (How upsetting is it for you?). The reliability and validity of this scale is established. ${ }^{8,9)}$

\section{Activation task}

Only normal healthy subjects participated in this study. According to Kubota et al., ${ }^{10)}$ healthy controls displayed greater activity in PFC with a letter VFT than with a category VFT under similar task performances, whereas patients with schizophrenia showed smaller activity in PFC with a letter VFT than with a category VFT. Therefore, we used a letter VFT in this study. VFT was conducted according to a previous study. ${ }^{11)}$ The procedure consists of a 30-s pre-task baseline, a 60-s task (text version), and a 30-s post-task baseline. All subjects were seated with their eyes open and were instructed to minimize head movements. During the pre- and post-task baseline periods, the subjects were instructed to repeat the vowels "a," "i," "u," "e," and "o" in Japanese. During VFT, the subjects were instructed to vocalize as many Japanese words as possible beginning with a designated syllable. The three initial syllables (1:/ma/, /ki/, /e/; 2: /to/, /u/,/se/; 3: $/$ te/, /i/, /ku/) were used in this order, and each syllable was changed every $20 \mathrm{~s}$ during the 60 -s task. During VFT, the subjects changed syllables as a counterbalance. The average total number of correct words stated was used to measure task performance.

\section{Near-infrared spectroscopy analysis}

Changes in the concentrations of oxygenated hemoglobin $[\mathrm{oxy}-\mathrm{Hb}]$ and deoxygenated $\mathrm{Hb}$ [deoxy-Hb] were measured using a two-channel near-infrared spectrometer
(PocketNIRS; DynaSense Inc., Hamamatsu, Japan) at wavelengths of $735 \mathrm{~nm}, 810 \mathrm{~nm}$, and $850 \mathrm{~nm}$. The [oxy- $\mathrm{Hb}$ ] and [deoxy- $\mathrm{Hb}$ ] were determined according to the modified Beer- Lambert law. ${ }^{12)}$ The two probes of the near-infrared spectrometer were attached with adhesive strips (DynaSense Inc.) and were centered at the Fp1 and Fp2 positions according to the international 10-20 electroencephalography system. The source-to-detector spacing of each probe was $3.0 \mathrm{~cm}$. NIRS provides a robust measure of hemodynamic changes within a $2-3 \mathrm{~cm}$ depth from the surface of the scalp. ${ }^{13)}$ The absorption of NIR was measured with a time resolution of $0.1 \mathrm{~s}$.

\section{Data Analysis}

Descriptive statistics were calculated for each variable. Two-sample $t$-tests were used to compare each variable between men and women.

We assessed the correlation between paranoia symptoms and [oxyHb] changes during VFT using partial correlation analyses (adjusted for gender, age, and task performance). Statistical analysis was performed using IBM SPSS Statistics software (version 19 for Windows; IBM Co., Armonk, NY, USA). The results are presented as mean \pm standard deviation, and a two-sided $p$ value of $<0.05$ was considered statistically significant.

\section{RESULTS}

\section{Characteristics of Subjects}

The mean age of the subjects ( 50 men and 28 women) was $20.0 \pm 1.1$ years (Table 1). The mean JPC scores are shown in Table 1. The frequency, conviction, and distress scores were $17.58 \pm 6.40,16.46 \pm 6.04$, and $16.85 \pm 7.33$, respectively. The task performance was $14.8 \pm 4.0$, and the mean left prefrontal cortex (LPFC) and right prefrontal cortex (RPFC) changes in [oxy-Hb] were $0.013 \pm 0.017 \mathrm{au}$ and $0.012 \pm 0.018 \mathrm{au}$, respectively (Table 1 ). There were no significant differences between the two groups in terms of age $(\mathrm{t}=-1.882, p=0.064)$, frequency scores $(\mathrm{t}=-0.927$, $p=0.357)$, conviction scores $(\mathrm{t}=-0.347, p=0.73)$, distress scores $(\mathrm{t}=1.073, p=0.286)$, task performance $(\mathrm{t}=-1.319$, $p=0.191), \quad[$ oxy-Hb] changes in LPFC $(\mathrm{t}=-1.153$, $p=0.253)$, and $[\mathrm{oxy}-\mathrm{Hb}]$ changes in RPFC $(\mathrm{t}=-1.405$, $p=0.164)$.

\section{Correlation between Paranoia Symptoms and Changes in the Concentration of Oxygenated Hemoglobin during the Task}

Table 2 lists the correlations between JPC and [oxy-Hb] 
Table 1. Subject demographics by gender

\begin{tabular}{lccc}
\hline & Total $(n=78)$ & Men $(n=50)$ & Women (n=28) \\
\hline Age (year) & $20.0 \pm 1.1$ & $20.12 \pm 1.24$ & $19.64 \pm 0.68$ \\
Frequency score of JPC & $17.58 \pm 6.40$ & $18.08 \pm 6.57$ & $16.68 \pm 6.1$ \\
Conviction score of JPC & $16.46 \pm 6.04$ & $16.64 \pm 6.14$ & $16.14 \pm 5.95$ \\
Distress score of JPC & $16.85 \pm 7.33$ & $16.18 \pm 7.22$ & 0.064 \\
Verbal fluency test & $14.8 \pm 4.0$ & $15.22 \pm 3.92$ & 0.357 \\
(oxy-Hb) changes in LPFC (au) & $0.013 \pm 0.017$ & $0.015 \pm 0.019$ & $18.04 \pm 7.51$ \\
(oxy-Hb) changes in RPFC (au) & $0.012 \pm 0.018$ & $0.014 \pm 0.02$ & $0.01 \pm 0.013$ \\
\hline
\end{tabular}

Values are presented as mean \pm standard deviation.

All tests were $p>0.05$.

JPC, Japanese version of the Paranoia Checklist; LPFC, left prefrontal cortex; RPFC, right prefrontal cortex; (oxy-Hb), concentrations of oxygenated hemoglobin.

Table 2. Correlation between JPC and changes in (oxy-Hb) during task performance

\begin{tabular}{lcccc}
\hline & LPFC & $p$ value & RPFC & $p$ value \\
\hline Frequency score of JPC & 0.000 & 0.997 & -0.006 & 0.962 \\
Conviction score of JPC & 0.019 & 0.868 & -0.048 & 0.681 \\
Distress score of JPC & 0.092 & 0.432 & 0.026 & 0.823 \\
\hline
\end{tabular}

All tests were $p>0.05$.

JPC, Japanese version of the Paranoia Checklist; LPFC, left prefrontal cortex; RPFC, right prefrontal cortex; (Oxy- $\mathrm{Hb})$, concentrations of oxygenated hemoglobin.

changes during the task. LPFC and RPFC [oxy-Hb] changes during VFT did not correlate significantly with frequency (LPFC: $\mathrm{r}=0.000, p=0.997$; RPFC: $\mathrm{r}=-0.006$, $p=0.962$ ), conviction (LPFC: $\mathrm{r}=0.019, p=0.868$; RPFC: $\mathrm{r}=$ $-0.048, \mathrm{p}=0.681$ ), and distress scores (LPFC: $\mathrm{r}=0.092$, $p=0.432$; RPFC: $\mathrm{r}=0.026, p=0.823$ ).

\section{DISCUSSION}

The main results of the present study show that the frequency, conviction, and distress scores of the symptoms of paranoia were not significantly correlated with the changes in LPFC and RPFC [oxy-Hb] during task performance. Several studies have demonstrated impairments of various areas of cognitive function in paranoid schizophrenia patients. ${ }^{3,14-17)}$ In addition, attention defects negatively affect other cognitive functions. ${ }^{18)}$ Because different hemodynamic response patterns are observed in the PFC sub-regions, according to the clinical stages of psychosis, distinct responses are expected in healthy subjects with paranoia who are not overt psychosis patients. ${ }^{19,20)}$ Moreover, our results are consistent with those reported by Hori et al., ${ }^{21)}$ who showed that paranoid ideation was not significantly correlated with prefrontal activation during VFT in healthy subjects. Therefore, our findings suggest that paranoia symptoms did not negatively influence prefrontal activation in healthy subjects. However, Hori et $a l .^{21)}$ reported that unusual perceptual experiences, social anxiety, and odd speech were correlated with prefrontal activation in healthy subjects. Therefore, it is necessary to examine in detail the relationship between prefrontal activation and other symptoms of schizophrenia in healthy subjects in future studies.

There are several limitations to this study. First, because it was cross-sectional, it precludes making firm conclusions regarding a causal relationship between prefrontal activation and symptoms of paranoia. Therefore, in the future, a longitudinal study may reveal the relationship between brain activation and degree of paranoia. Second, the NIR spectrometer was equipped with only two channels; therefore, only limited areas of the brain were measured. Future studies using a multichannel instrument are required. Third, loss of sleep causes a significant increase in paranoia scores. ${ }^{22)}$ This was not taken into account here, but it will be in the future study.

In conclusion, the present results show that in the symptoms of paranoia did not negatively affect prefrontal activation during performance of a task in healthy subjects.

\section{Acknowledgments}

I would like to thank all the subjects who participated in this study.

\section{REFERENCES}

1. Andreasen NC, Flaum M. Schizophrenia: the characteristic symptoms. Schizophr Bull 1991;17:27-49.

2. Loh M, Rolls ET, Deco G. A dynamical systems hypothesis of schizophrenia. PLoS Comput Biol 2007;3:e228.

3. Williams LM, Das P, Liddell BJ, Olivieri G, Peduto AS, David AS, et al. Fronto-limbic and autonomic disjunctions to negative emotion distinguish schizophrenia subtypes. Psychiatry Res 2007; 155:29-44.

4. Freeman D, Garety PA, Bebbington PE, Smith B, Rollinson $\mathrm{R}$, Fowler $\mathrm{D}$, et al. Psychological investigation of the structure of paranoia in a non-clinical population. $\mathrm{Br} J$ Psychiatry 2005;186:427-435. 
5. Ellett L, Lopes B, Chadwick P. Paranoia in a nonclinical population of college students. J Nerv Ment Dis 2003;191: 425-430.

6. McKay R, Langdon R, Coltheart M. The persecutory ideation questionnaire. J Nerv Ment Dis 2006; 194:628-631.

7. Strangman G, Culver JP, Thompson JH, Boas DA. A quantitative comparison of simultaneous BOLD fMRI and NIRS recordings during functional brain activation. Neuroimage 2002;17:719-731.

8. Yamauchi T, Sudo A, Tanno Y. Reliability and validity of the Japanese Version of Paranoia Checklist. Jpn J Pers 2007; 16:114-116.

9. Yamauchi T, Sudo A, Tanno Y. Factor structure and validity of Japanese-version Paranoia Checklist. Jpn J Pers 2009; 17:182-193.

10. Kubota Y, Toichi M, Shimizu M, Mason RA, Coconcea CM, Findling RL, et al. Prefrontal activation during verbal fluency tests in schizophrenia--a near-infrared spectroscopy (NIRS) study. Schizophr Res 2005;77:65-73.

11. Takizawa R, Kasai K, Kawakubo Y, Marumo K, Kawasaki $\mathrm{S}$, Yamasue $\mathrm{H}$, et al. Reduced frontopolar activation during verbal fluency task in schizophrenia: a multi-channel nearinfrared spectroscopy study. Schizophr Res 2008;99:250262.

12. Mizuno T, Watanabe T, Iwai H, Shikayama T, Miwa M, Yamashita $\mathrm{Y}$, et al. High-speed sampling measurements of tissue oxygenation by the portable near-infrared monitoring system. Iryou kikigaku 2010;80:189-195.

13. Okada E, Delpy DT. Near-infrared light propagation in an adult head model. II. Effect of superficial tissue thickness on the sensitivity of the near-infrared spectroscopy signal. Appl Opt 2003;42:2915-2922.

14. Müller W, Haffelder G, Schlotmann A, Schaefers AT, Teuchert-Noodt G. Amelioration of psychiatric symptoms through exposure to music individually adapted to brain rhythm disorders - a randomised clinical trial on the basis of fundamental research. Cogn Neuropsychiatry 2014;19: 399-413.

15. Pankow A, Knobel A, Voss M, Heinz A. Neurobiological correlates of delusion: beyond the salience attribution hypothesis. Neuropsychobiology 2012;66:33-43.

16. Pinkham AE, Brensinger C, Kohler C, Gur RE, Gur RC. Actively paranoid patients with schizophrenia over attribute anger to neutral faces. Schizophr Res 2011;125:174-178.

17. Gromann PM, Heslenfeld DJ, Fett AK, Joyce DW, Shergill SS, Krabbendam L. Trust versus paranoia: abnormal response to social reward in psychotic illness. Brain 2013;136:1968-1975.

18. Hooker CI, Benson TL, Gyurak A, Yin H, Tully LM, Lincoln SH. Neural activity to positive expressions predicts daily experience of schizophrenia-spectrum symptoms in adults with high social anhedonia. J Abnorm Psychol 2014;123:190-204.

19. Koike S, Takizawa R, Nishimura Y, Takano Y, Takayanagi $\mathrm{Y}$, Kinou M, et al. Different hemodynamic response patterns in the prefrontal cortical sub-regions according to the clinical stages of psychosis. Schizophr Res 2011;132:54-61.

20. Moritz S, Laudan A. Attention bias for paranoia-relevant visual stimuli in schizophrenia. Cogn Neuropsychiatry 2007; 12:381-390.

21. Hori H, Nagamine M, Soshi T, Okabe S, Kim Y, Kunugi H. Schizotypal traits in healthy women predict prefrontal activation patterns during a verbal fluency task: a nearinfrared spectroscopy study. Neuropsychobiology 2008;57: 61-69.

22. Kahn-Greene ET, Killgore DB, Kamimori GH, Balkin TJ, Killgore WD. The effects of sleep deprivation on symptoms of psychopathology in healthy adults. Sleep Med 2007; 8:215-221. 